\title{
Imaging Multi-Protein Complex Formation in the Autophagy Pathway
}

\author{
L.J. Kraft, M. Kang, J. Greer, K. Drake, and A.K. Kenworthy \\ Department of Molecular Physiology and Biophysics, Vanderbilt University, Nashville, TN 37212
}

With the massive influx of information about protein-protein interactions coming from bioinformatics and proteomics analyses [1], the bottleneck has shifted towards a more thorough characterization of these interactions within the context of the living cell. As a result of recent advances in live-cell imaging, it has become possible to quantitatively investigate the spatial and temporal regulation of the interactions of green fluorescent protein (GFP) tagged proteins in the complex environment of the living cell [2].

One example of an intracellular pathway whose function depends on the coordinated effort of a network of interacting proteins is macroautophagy [3]. The process of macroautophagy is activated in response to stress signals such as low nutrient availability. Up-regulation of this pathway leads to the capture of cytosolic materials in a double membrane structure termed the autophagosome that is subsequently trafficked to the lysosome for degradation and recycling. The protein microtubule protein 1 light chain 3 (LC3) plays a central role in the autophagy pathway, and through a set of ubiquitin-like reactions LC3 becomes associated with developing autophagosomes. LC3 exists in both a soluble form termed LC3-I, and a tightly membrane associated form termed LC3-II localized primarily on developing autophagosomes [4]. LC3 has become a widely used tool to monitor autophagy pathway flux, and when fused to GFP, to selectively highlight autophagosomal structures $[3,5]$.

Recently, we obtained evidence from diffusion-based measurements that LC3-I may be present as part of a large multi-protein complex, not only in the cytoplasm, but also in the nucleus [6]. This finding illustrates the power of live cell imaging approaches to identify novel features of the autophagy pathway, and also raises the possibility that the complexes we identified may correspond to those implied in a large-scale proteomics screen of autophagy interacting proteins [1]. To address this question, we are developing a combined diffusion and FRET-based approach to probe for multiprotein complexes containing LC3 in living cells.

To establish methods to probe for complex formation, we have begun to investigate the interactions of LC3 with Atg4B. Atg4B, a cysteine protease, is known to play two important roles in both the lipidation and delipidation of LC3 [4]. A mutant form of Atg4B, Atg4 ${ }^{\mathrm{C} 74 \mathrm{~A}}$, has previously been shown to sequester LC3 in the cytosol and prevent LC3 from binding to autophagosomes [7]. In support of these findings, we recently showed co-overexpression of LC3 and Atg $4 \mathrm{~B}^{\mathrm{C} 74 \mathrm{~A}}$ shifts the localization of LC3 out of the nucleus [6]. Therefore, the known interaction of LC3 and Atg4B ${ }^{\mathrm{C} 74 \mathrm{~A}}$ serves as a good positive control for development of FRET and diffusion-based assays to test for protein complex formation in the autophagy pathway.

To test for a direct interaction between LC3 and $\mathrm{Atg} 4 \mathrm{~B}^{\mathrm{C} 74 \mathrm{~A}}$ in vivo, we used acceptor photobleaching FRET microscopy. Our preliminary studies show detectable FRET between Cerulean-LC3 and Venus-Atg4 $\mathrm{B}^{\mathrm{C} 74 \mathrm{~A}}$ in the cytoplasm (Fig. 1), and no FRET between negative control Cerulean and Venus-Atg4 $\mathrm{B}^{\mathrm{C} 74 \mathrm{~A}}$. This suggests LC3 and Atg $4 \mathrm{~B}^{\mathrm{C} 74 \mathrm{~A}}$ directly interact in live cells, in support of previous in vitro biochemical studies [7]. 
In order to determine if $\mathrm{Atg} 4 \mathrm{~B}^{\mathrm{C} 74 \mathrm{~A}}$ behaves like a freely diffusing monomer or instead shows evidence for being part of a larger complex, we performed confocal FRAP measurements of individually overexpressed Venus-LC3, Venus-Atg4B ${ }^{\mathrm{C} 74 \mathrm{~A}}$, and Venus. Similar to LC3, the diffusion of $A \operatorname{tg} 4 \mathrm{~B}^{\mathrm{C} 74 \mathrm{~A}}$ is much slower than anticipated for a freely diffusing monomer. Next, we asked whether the mobility of LC3 is altered when co-overexpressed and bound to $\operatorname{Atg} 4 \mathrm{~B}^{\mathrm{C} 74 \mathrm{~A}}$. Surprisingly, our preliminary studies suggest that the mobility of LC3 is unchanged upon cooverexpression with $A \operatorname{tg} 4 \mathrm{~B}^{\mathrm{C} 74 \mathrm{~A}}$. Together with our FRET study, these results indicate $\mathrm{Atg} 4 \mathrm{~B}^{\mathrm{C} 74 \mathrm{~A}}$ and LC3 may both be part of a large multi-protein complex.

To extend these findings, we plan to perform FCS measurements to specifically test for the presence of multiple diffusing species of LC3 and Atg4B ${ }^{\mathrm{C} 74 \mathrm{~A}}$, and to investigate the dynamics of the LC3$\mathrm{A} \operatorname{tg} 4 \mathrm{~B}^{\mathrm{C} 74 \mathrm{~A}}$ interaction using a combined FRET and FRAP approach called photoquenching FRET [8].

In future studies, we will apply these methods to other known LC3 interacting proteins in the autophagy pathway, as well as determine how these interactions may change under starvation conditions. These types of fundamental measurements of live-cell protein diffusion and complex formation will be useful for systems-biology modeling and ultimately for developing rational strategies to modulate the autophagy pathway with new drugs.

\section{References}

[1] C. Behrends, et al., Nature 68-76 (2010) 466.

[2] J. Lippincott-Schwartz, et al., Nat Rev Mol Cell Biol 444-456 (2001) 2.

[3] Z. Yang, and D.J. Klionsky, Nat Cell Biol 814-822 (2010) 12.

[4] T. Kirisako, et al., J Cell Biol 263-76 (2000) 151.

[5] N. Mizushima, et al., Cell 313-26 (2010) 140.

[6] K.R. Drake, et al., PloS one e9806 (2010) 5.

[7] N. Fujita, et al., Mol Biol Cell 4651-4659 (2008) 19.

[8] I.A. Demarco, et al., Nat Methods 519 (2006) 3.

[9] This work is supported by R01 GM073846 and NSF/DMS 0970

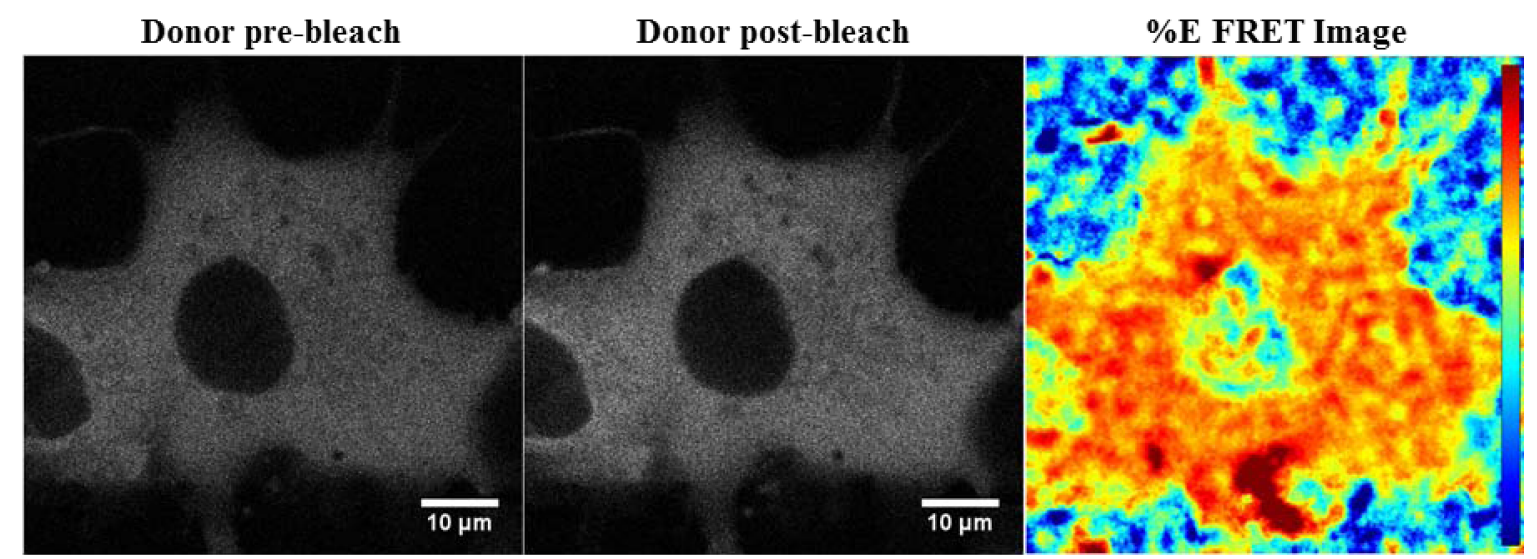

FIG 1. Using the acceptor photobleaching technique we detect FRET between Cerulean-Atg4B ${ }^{\mathrm{C} 74 \mathrm{~A}}$ and Venus-LC3 in the cytoplasm and nucleus of COS-7 cells. 